\title{
Cluster headache management and beyond
}

\section{Paolo Martelletti}

To cite this article: Paolo Martelletti (2015) Cluster headache management and beyond, Expert Opinion on Pharmacotherapy, 16:10, 1411-1415

To link to this article: http://dx.doi.org/10.1517/14656566.2015.1052741

\section{曲 Published online: 01 Jun 2015.}

Submit your article to this journal

Џلll Article views: 248

Q View related articles $\sqsubset$

View Crossmark data ¿ 


\section{EXPERT OPINION}

1. Cluster headache, very popular but still underdiagnosed

2. Early therapeutic management needed, but delayed diagnosis frustates it

3. Transitional therapy is necessary, but beware of the carry-over phenomenon

4. Preventative treatment as a cornerstone

5. Beyond the reliable pharmacological triad

\section{informa}

\section{Cluster headache management and beyond}

\author{
Paolo Martelletti \\ Sapienza University, Department of Clinical and Molecular Medicine, Rome, Italy
}

The therapeutic management of cluster headache is based on a very stable triad of drugs. Acute treatment has, in subcutaneous sumatriptan, its gold standard if compared to pure oxygen or indomethacin. Preventative treatment is based on verapamil at high doses ( $\geq 360 \mathrm{mg}$ ) and is a gold standard if compared to lithium carbonate or topiramate. Transitional treatments, based on the short-term use of corticosteroids with either systemic or local administration (GON), can be useful for the suppression of most resistant cluster periods, but with a well-known carry-over phenomenon related to the length of the cluster period itself. The role of invasive or noninvasive neuromodulation approaches must still be determined on a large scale; therefore, its use is not recommended as of yet. Lifestyle changes, including alcohol avoidance during the active phase of the disease, sleep hygiene and use of vasodilation drugs, should be carefully considered and the patients should be fully informed.

Keywords: cluster headache, corticosteroids, GON block, neurostimulation, oxygen, sumatriptan, verapamil

Expert Opin. Pharmacother. (2015) 16(10):1411-1415

\section{Cluster headache, very popular but still underdiagnosed}

Cluster headache $(\mathrm{CH})$ is the most common of the trigeminal autonomic cephalalgias (TACs) [1]. CH is a strictly lateralized and devastating periorbital headache, characterized with a very severe, excruciating pain and the presence of ipsilateral cranial autonomic symptoms, such as conjunctival injection, lacrimation and rhinorrhea. Lifetime prevalence is $1 / 1000$ and a progressive reduction of the male/ female ratio over the past decades has been demonstrated and is now 2.1:1 [2] The peculiar timing over the day ( $1-8$ attacks $)$, in the entire active cluster period lasting 60 - 90 days, and in the span of the year particularly with the seasonal changes, renders this headache unique, very easy to diagnose and differentiate from other TACs [1]. Nevertheless, only $1 / 3$ of these patients received the diagnosis of $\mathrm{CH}$, with a diagnostic delay of 5.3 years and more than $2 / 3$ of them never received any treatment. The most frequent clinical errors behind this delay are trigeminal neuralgia, migraine without aura, headache attributed to idiopathic intracranial hypertension, tension-type headache, depression, dental and sinus pathologies [3]. The strict laterality of pain, even though considered a clinical marker, might shift sides in a minority of patients, either within the bouts of the same cluster period or between the bouts of different cluster periods [4].

The natural history of this illness, which generally begins between 20/30 years of age, shows seasonal cluster periods with an unpredictable variability of their circaannual rhythms. This leads to overestimate the effect of the administered therapy showing often a spontaneous prolonged remission. Only $10 \%$ of patients with episodic cluster headache $(\mathrm{ECH})$ develop a chronic form $(\mathrm{CCH})$ (> 180 days/ year) and even fewer develop refractory or medically resistant chronic cluster headache $(\mathrm{rCCH})[5]$. 
The strong evidence supporting the pathophysiology of $\mathrm{CH}$ revolves around the suggestion of a posterior hypothalamic dysfunction (anatomic, endocrine, genetic, neuro-immunological and functional imaging). Scientists are therefore oriented toward its involvement [6] even if this solid hypothesis has been recently re-interpreted as an epiphenomenon [7].

Rare cases of secondary cluster-like headaches are ascribable to pituitary tumors, fungal cysts or dissection of the internal carotid artery and are extremely challenging for the clinician, also the expert one [8].

\section{Early therapeutic management needed, but delayed diagnosis frustates it}

$\mathrm{CH}$ therapeutic management varies according to the three forms previously cited (ECH, $\mathrm{CCH}, \mathrm{rCCH})$.

Acute, transitional and preventative treatments can be applied to both ECH and CCH. The gold standard for the acute treatment of $\mathrm{CH}$ is subcutaneous sumatriptan, able to dissolve the attack in a handful of minutes [9-11]. Patients with previous ischemic and cardio-cerebral pathologies must be excluded from such therapy. Other sumatriptan or zolmitriptan formulations do not show, from a clinical point of view, the needed rapidity and completeness of action despite reported scientific evidence [12-16].

Indomethacin may, in some cases, be a helpful alternative even if there is no clear-cut evidence of its efficacy for $\mathrm{CH}$. This drug is generally not recommended by international guidelines, and should not be used in the presence of arterial hypertension or gastric bleeding [17].

Pure oxygen inhalation at a flow of $7-15 \mathrm{l} / \mathrm{min}$, delivered by facemask, possibly with reservoir for no more than $20 \mathrm{~min}$, is safer and, on its own, showed a very meaningful effect on $\mathrm{CH}$ attack. The main concern is that, in some patients, it may delay the attacks rather than abort them. Unfortunately, oxygen presents applicative limitations in terms of portability $[18,19]$.

Hyperbaric oxygen has been reported as a rescue alternative in chronic $\mathrm{CH}$ where other options resulted inopportune, but its intrinsic limits sum up with the lack of accessibility [20].

Recently, vagal nerve stimulation (VNS) has been proposed for the treatment of the attack and for prophylaxis, both in episodic and chronic $\mathrm{CH}$. The initial experience indicates that noninvasive vagus nerve stimulation may be practical and effective as an acute and preventive treatment in $\mathrm{CCH}$. This Class IV evidence technique should be confirmed by randomized controlled trials (RCTs) [21].

\section{Transitional therapy is necessary, but beware of the carry-over phenomenon}

In clinical situations showing a cluster period lasting up to $4-5$ months with a risk of chronicization, transitional block of the greater occipital nerve with long-acting corticosteroids can be considered. This option may lead to a clinical amelioration and should be repeated cyclically. The limitation of this approach resides in the nonfamiliarity or the reluctance of the headache expert toward this mini-invasive but very easy technique [22].

Cortisone per os (prednisone $1 \mathrm{mg} / \mathrm{kg}$ with a tapering regimen) is still widely used but inadvisable since it causes the carry-over phenomenon of the cluster or, even worse, a violent rebound.

\section{Preventative treatment as a cornerstone}

The gold standard of preventative treatment is still verapamil taken at medium-high doses (from $360 \mathrm{mg} /$ day up to $960 \mathrm{mg}$ ). The starting dose is always $360 \mathrm{mg}$ ex abrupto for patients with a clear absence of bradycardia $(<50 \mathrm{bpm})$, hypotension $(<100 / 60 \mathrm{mmHg}$ ) or other cardiac arrhythmias (atrioventricular block). Uncomfortable adverse effects of ankle edema [23] are tolerated by patients considering verapamil's fast action, less than $72 \mathrm{~h}$ to crush the bouts.

Lithium is less handy than verapamil. It needs a longer latency period (up to 10 - 15 days) because of the need for progressive titration up to $900-1200 \mathrm{mg} /$ day and a burdensome bimonthly evaluation of lithium levels $(0.8-1.2 \mu \mathrm{mol} / \mathrm{l})$; referential parameter for an effective therapeutic action, but with important inter-individual variations. Serious side effects are hypothyroidism, diabetes insipidus and mental confusion.

The use of topiramate at optimal dosage (within 100 and $200 \mathrm{mg} /$ day) is strongly limited by its serious side effects (slow tritation, serious cognitive impairment) and contraindications (glaucoma, kidney stones, severe temporary weight loss and irreversible drop of visual acuity).

Melatonin may be included as preventative treatment on the basis of a small pilot RCT that demonstrated its clinical relevance in $\mathrm{CH}$ [24].

Other supposed preventative therapies with gabapentin, baclofen, levetiracetam, pizotifen, sodium valproate, testosterone, misoprostol, civamide, warfarin, candesartan, acupunture or other drugs no longer available, like dihydroergotamine and methysergide, are not clinically relevant.

The mentioned preventative treatments must be started at the first sight of activation/re-activation of the cluster period and must be continued for a period equal to 1.5 of the historical length of the patient's single cluster. Shorter treatments might produce a temporal shift of the active period, even if this re-firing has a lower intensity. Therefore, the most appropriate semantic definition of the preventative therapy should be "control/suppression therapy" of the cluster period.

\section{Beyond the reliable pharmacological triad}

A hypothetical tripod for the therapy of $\mathrm{CH}$ can be summarized in sumatriptan, verapamil and corticosteroids. New therapeutic opportunities are now enriching the literature on the subject of migraines. These new compounds, monoclonal 


\section{Management road for cluster headache}

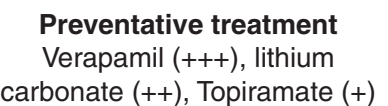

Preventative treatment carbonate $(++)$, Topiramate $(+)$

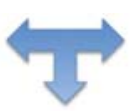

Acute treatment Sumatriptan $6 \mathrm{mg} \mathrm{sc}$, pure oxygen, indomethacin

Transitional treatment

Corticosteroids (oral, GON, iv, im)
Neuromodulation treatment VNS, SPG, ONS, SCS
Lifestyle changes

Avoidance of alcohol, sleep changes and vasodilation drugs

Figure 1. Comprehensive management of cluster headache.

antibodies against calcitonin gene-related peptide, could soon be available as preventative treatment of migraine [25] and, hopefully, for $\mathrm{CH}$ as well.

Furthermore, another important topic to be considered is patients' knowledge regarding possible triggers. Patients should be educated to avoid any substance that, in the active phase of $\mathrm{CH}$, might work as a strong activator of the bouts, like alcohol, nitroderivative drugs used for the treatment of angina pectoris, and phosphodiesterase inhibitors used for erectile dysfunction.

Therefore, only with better education of physicians at the different care levels (general practitioners, neurologists) and of patients, an early diagnosis is possible, since an adequate treatment of $\mathrm{CH}$ cuts the risk of chronicization to a minimum. A practical concise scheme for the management of $\mathrm{CH}$ is summarized in Figure 1.

It goes without saying that the introduction of new, more manageable and efficacious preventative drugs might stop the therapeutic alarm that often hovers around this impressive and psychologically destabilizing painful pathology. These drugs may also reduce the chronicization rate often linked to an inefficacy $a b$ initio of the therapeutic preventative opportunities now available.

Another point to be mentioned regards the new invasive and noninvasive neuromodulation approaches for $\mathrm{CCH}$. Despite promising new data, the European Headache
Federation recommends caution in using these techniques [26] because only few controlled studies have, as yet, been carried out. It is important to avoid the disillusion produced in $\mathrm{CCH}$ patients when, more than 10 years ago, the scientific community celebrated as decisive, an extremely invasive and harmful therapeutic proposal [27], now abandoned [26,28].

Lastly, going beyond the actual management of $\mathrm{CH}$, VNS seems to be promising but is still classified at Class IV evidence [21], while sphenopalatine ganglion stimulation shows contrasting evidence [29,30] that needs to be confirmed and validated through RCTs.

On the other hand, new hopes rely on the future application of an RCT efficacy study of LY2951742 in episodic $\mathrm{CH}$. This drug, if approved, could change the management of $\mathrm{CH}$ rendering it very easy to use through a subcutaneous administration every 30 days [31].

\section{Declaration of interest}

The author has no relevant affiliations or financial involvement with any organization or entity with a financial interest in or financial conflict with the subject matter or materials discussed in the manuscript. This includes employment, consultancies, honoraria, stock ownership or options, expert testimony, grants or patents received or pending, or royalties. 


\section{Bibliography}

Papers of special note have been highlighted as either of interest $(\bullet)$ or of considerable interest

$(\bullet \bullet)$ to readers.

1. Headache Classification Committee of the International Headache Society. The International Classification of Headache Disorders, 3rd edition (beta version). Cephalalgia 2013;33:629-808

2. Manzoni GC. Gender ratio of cluster headache over the years: a possible role of changes in lifestyle. Cephalalgia 1998;18(3):138-42

3. Voiticovschi-Iosob C, Allena M, De Cillis I, et al. Diagnostic and therapeutic errors in cluster headache: a hospital-based study. J Headache Pain 2014;15:56-doi: 10.1186/1129-2377-1556

4. Sjaastad O, Saunte C, Fredriksen TA. Bilaterality of cluster headache. An hypothesis. Cephalalgia 1985;5(1):55-8

5. Mitsikostas DD, Edvinsson L, Jensen RH, et al. Refractory chronic cluster headache: a consensus statement on clinical definition from the European Headache Federation. J Headache Pain 2014;15:79

- An important official document on the definition of refractory Chronic Cluster Headache.

6. Goadsby P. Trigeminal autonomic cephalalgias. Continuum (Minneap Minn) 2012;18(4):883-95

7. Holle D, Obermann M. Cluster headache and the hypothalamus: causal relationship or epiphenomenon? Expert Rev Neurother 2011;11:1255-63

8. Edvardsson B. Symptomatic cluster headache: a review of 63 cases. Springerplus 2014;3:64

9. The Sumatriptan Cluster Headache Study Group. Treatment of acute cluster headache with sumatriptan. N Engl J Med 1991;325:322-6

10. Ekbom K, Krabbe A, Micelli G, et al. Cluster headache attacks treated for up to three months with subcutaneous sumatriptan $(6 \mathrm{mg})$. Cephalalgia 1995; 15:230-6

11. Lionetto L, Negro A, Casolla B, et al. Sumatriptan succinate: pharmacokinetics of different formulations in clinical practice. Expert Opin Pharmacother 2012;13(16):2369-80
12. van Vliet JA, Bahra A, Martin V, et al. Intranasal sumatriptan in cluster headache: randomized placebo-controlled double-blind study. Neurology 2003;60:630-3

13. Cittadini E, May A, Straube A, et al. Effectiveness of intranasal zolmitriptan in acute cluster headache: a randomized, placebo-controlled, double-blind crossover study. Arch Neurol 2006;63:1537-42

14. Rapoport AM, Mathew NT, Silberstein SD, et al. Zolmitriptan nasal spray in the acute treatment of cluster headache: a double-blind study. Neurology 2007;69:821-6

15. Lionetto L, Casolla B, Mastropietri F, et al. Pharmacokinetic evaluation of zolmitriptan for the treatment of migraines. Expert Opin Drug Metab Toxicol 2012;8(8):1043-50

16. Law S, Derry S, Moore RA. Triptans for acute cluster headache.

Cochrane Database Syst Rev 2013;7:CD008042

- A comprehensive review on the drug acute treatment of Cluster Headache.

17. VanderPluym J. Indomethacin-responsive headaches. Curr Neurol Neurosci Rep 2015;15:516

18. Cohen AS, Burns B, Goadsby PJ. High-flow oxygen for treatment of cluster headache: a randomized trial. JAMA 2009;302(22):2451-7.doi: 10.1001/ jama.2009.1855

19. Petersen AS, Barloese MC, Jensen RH. Oxygen treatment of cluster headache: a review. Cephalalgia 2014;34(13):1079-87

20. Di Sabato F, Rocco M, Martelletti P, et al. Hyperbaric oxygen in chronic cluster headaches: influence on serotonergic pathways. Undersea Hyperb Med 1997;24:117-22

21. Nesbitt AD, Marin JC, Tompkins E, et al. Initial use of a novel noninvasive vagus nerve stimulator for cluster headache treatment. Neurology 2015;84(12):1249-53

22. Lambru G, Abu Bakar N, Stahlhut L, et al. Greater occipital nerve blocks in chronic cluster headache: a prospective open-label study. Eur J Neurol 2014;21(2):338-43

- This paper shows how Cluster Headache can be approached by GON blockade.

23. Elliott WJ, Ram CV. Calcium channel blockers. J Clin Hypertens (Greenwich) 2011;13(9):687-9

24. Leone M, D'Amico D, Moschiano F, et al. Melatonin versus placebo in the prophylaxis of cluster headache: a double-blind pilot study with parallel groups. Cephalalgia 1996;16:494-6

25. Dodick DW, Goadsby PJ, Spierings EL, et al. Safety and efficacy of LY2951742, a monoclonal antibody to calcitonin gene-related peptide, for the prevention of migraine: a phase 2, randomised, double-blind, placebo-controlled study. Lancet Neurol 2014;13(9):885-92

26. Martelletti P, Jensen RH, Antal A, et al. Neuromodulation of chronic headaches: position statement from the European Headache Federation. J Headache Pain 2013;14:86

-. An extremely updated official statement summarizing the state of the art on neuromodulation of chronic headaches.

27. Leone M, Franzini A, Bussone G. Stereotactic stimulation of posterior hypothalamic gray matter in a patient with intractable cluster headache. N Engl J Med 2001;345(19):1428-9

28. Fontaine D, Lazorthes Y, Mertens P, et al. Safety and efficacy of deep brain stimulation in refractory cluster headache: a randomized placebocontrolled double-blind trial followed by a 1-year open extension. J Headache Pain 2010;11(1):23-31

29. Schoenen J, Jensen RH, Lantéri-Minet M, et al. Stimulation of the sphenopalatine ganglion (SPG) for cluster headache treatment. Pathway $\mathrm{CH}$ 1: a randomized, sham-controlled study. Cephalalgia 2013;33(10):816-30

30. Bendersky DC, Hem SM, Yampolsky CG. Unsuccessful pulsed radiofrequency of the sphenopalatine ganglion in patients with chronic cluster headache and subsequent successful thermocoagulation. Pain Pract 2015; doi: 10.1111/papr.12288. [Epub ahead of print] 
31. ClinicalTrial.gov. A study of

LY2951742 in participants with episodic cluster headache. Available from: https:// clinicaltrial.gov/ct2/show/NCT02397473 [Last accessed 7 May 2015]

\section{Affiliation}

Paolo Martelletti

Sapienza University, Department of Clinical and Molecular Medicine, Rome, Italy

Tel: +3906337751 10;

Fax: +3906337751 11;

E-mail: paolo.martelletti@uniroma1.it 